\title{
EDITORIAL
}

\section{Fuch's Heterochromic Cyclitis}

Despite the enormous literature on Fuch's heterochromic cyclitis, we are little further forward in understanding its pathogenesis. The fact that many eyes become quiet after cataract extraction has not been satisfactorily explained but would appear to implicate the lens somehow in its perpetuation but not necessarily the initiating mechanism(s). In its classic form, it is easy to spot and is worth recognising since the patient is then spared prolonged and ineffective courses of topical steroids and mydriatrics. There are many unresolved questions about Fuch's-how many features are necessary to make the diagnosis? Do a few pigmented KP or pigment spots on the lens or other types of cataract apart from posterior subcapsular lens opacities invalidate the diagnosis?

Why do the anterior segment inflammatory signs in Fuch's not respond to topical steroids like other forms of anterior uveitis? Is it possible that other processes apart from inflammation, such as ischaemia are involved? The articles by Nicholas Jones in this issue describe very well the spectrum of signs seen in this disorder but whether all ophthalmologists would categorise all the patients diagnosed by him as Fuchs' cyclitis is debatable.

Does it really matter whether the disease is called Fuchs' cyclitis or chronic iritis? Probably not for the patient requiring cataract surgery-the posterior segment is not involved so that peroperative systemic steroid cover is unnecessary, just like other forms of isolated anterior uveitis, and intraocular lenses can safely be implanted. The surgeon, however, will be cheered by the knowledge that the pupil will be mobile, and alert to the possibility of post-operative pressure problems. Management of the glaucoma is similar in Fuchs' and other anterior uveitis. $\mathrm{Mr}$ Jones found a high failure rate for conventional fistulising surgery in a small group, not a universal finding by others. However, where it does matter is in routine management and in the advice given to the patient. Many are young, including children, and if not correctly identified could either be given no treatment when it is really needed so that they develop complications such as posterior synechiae or have years of useless steroid drops which may hasten the development of cataract and glaucoma. Patients with Fuchs' type also need far less in the way of routine follow-up in outpatients than those with anterior uveitis.

So what are the minimal diagnostic criteria? It depends on which study you want to follow. Uniocular disease, non-pigmented stellate KP especially over the upper posterior surface of the cornea which do not disappear with topical steroid therapy, absence of posterior synechiae, posterior subcapsular lens opacities and a fibrillar change in the anterior vitreous we would probably all agree on. Heterochromia, most often seen in light brown irides is a bonus when present but many patients with chronic anterior uveitis develop a 'moth-eaten' appearance to their iris due to stromal changes. In most cases, the iris and angle vessel prominence is due to this atrophy and not to neovascularisation although this can also occur rarely according to the literature.

Bilateral disease can occur, the patients can have inflammatory exacerbations which do respond to topical steroids and they can develop vitreous opacification as well. Are snowballs or snowbanks allowed-we would put these into the pars planitis group, which has a different set of problems and requires different management strategies. Heterochromia is not necessary, nor are abnormal vessels in the angle. Pigmented KP and posterior synechise prior to surgery largely exclude the diagnosis. What about the patient who has all the right signs including resistance to topical steroids but develops a few pigmented KP_do we still call him Fuchs' type? We agree with Nicholas Jones-it is likely that Fuchs' cyclitis is not one disease entity but a spectrum of disorders and will no doubt continue to puzzle us for years to come.

Susan Lightman and Hamish Towler 